PROCEEDINGS OF THE

AMERICAN MATHEMATICAL SOCIETY

Volume 128, Number 12, Pages 3621-3628

S 0002-9939(00)05638-0

Article electronically published on June 6, 2000

\title{
LAMÉ DIFFERENTIAL EQUATIONS AND ELECTROSTATICS
}

\author{
DIMITAR K. DIMITROV AND WALTER VAN ASSCHE
}

(Communicated by Hal L. Smith)

\begin{abstract}
The problem of existence and uniqueness of polynomial solutions of the Lamé differential equation

$$
A(x) y^{\prime \prime}+2 B(x) y^{\prime}+C(x) y=0,
$$

where $A(x), B(x)$ and $C(x)$ are polynomials of degree $p+1, p$ and $p-1$, is under discussion. We concentrate on the case when $A(x)$ has only real zeros $a_{j}$ and, in contrast to a classical result of Heine and Stieltjes which concerns the case of positive coefficients $r_{j}$ in the partial fraction decomposition $B(x) / A(x)=\sum_{j=0}^{p} r_{j} /\left(x-a_{j}\right)$, we allow the presence of both positive and negative coefficients $r_{j}$. The corresponding electrostatic interpretation of the zeros of the solution $y(x)$ as points of equilibrium in an electrostatic field generated by charges $r_{j}$ at $a_{j}$ is given. As an application we prove that the zeros of the Gegenbauer-Laurent polynomials are the points of unique equilibrium in a field generated by two positive and two negative charges.
\end{abstract}

\section{INTRODUCTION}

Let $\left\{P_{n}^{(\alpha, \beta)}(x)\right\}_{n=0}^{\infty}$ be the classical Jacobi polynomials, orthogonal on $[-1,1]$ with respect to the weight function $(1-x)^{\alpha}(1+x)^{\beta}$. One of the reasons for the interest in the zeros of $P_{n}^{(\alpha, \beta)}(x)$ is that they are the points of equilibrium of $n$ free unit charges in $(-1,1)$ in the field generated by two fixed charges $(\alpha+1) / 2$ at 1 and $(\beta+1) / 2$ at -1 , where the charges repel each other according to the law of logarithmic potential. This means that the charges are not point charges but are distributed along infinite straight wires perpendicular to the real axis [11]. This beautiful interpretation is due to Stieltjes [13, 14, 15, who proved that the energy of the field has a local minimum at the zeros of the Jacobi polynomial of degree $n$. Szegő [16, Section 6.7] proved that the energy has a unique minimum, thus establishing the stability of the equilibrium. The basic idea of Stieltjes' and Szegö's proofs lies in the following:

Received by the editors February 22, 1999.

1991 Mathematics Subject Classification. Primary 34C10, 33C45; Secondary 34B30, 42C05.

Key words and phrases. Lamé differential equation, electrostatic equilibrium, Laurent polynomials, Gegenbauer polynomials.

The research of the first author is supported by the Brazilian Science Foundations FAPESP under Grant 97/6280-0 and CNPq under Grant 300645/95-3.

The second author is a Research Director of the Belgian Fund for Scientific Research (FWO-V). Research supported by FWO research project G.0278.97. 
Theorem A ([16, Theorem 4.2.2]). Let $\alpha>-1$ and $\beta>-1$. The differential equation

$$
\left(1-x^{2}\right) y^{\prime \prime}+(\beta-\alpha-(\alpha+\beta+2) x) y^{\prime}+\gamma y=0,
$$

where $\gamma$ is a parameter, has a polynomial solution not identically zero if and only if $\gamma$ has the form $n(n+\alpha+\beta+1), n=0,1, \ldots$ Moreover, if $\gamma=n(n+\alpha+\beta+1)$, the polynomials $c P_{n}^{(\alpha, \beta)}(x)$, where $c$ is any constant, are solutions of this equation, and there are no other polynomial solutions.

Stieltjes investigated the following more general situation: given $p+1$ positive charges $r_{j}$ at $a_{j}, a_{0}<\ldots<a_{p}$, find all possible equilibrium locations $x_{k}$ of $n$ free unit charges. As it is shown in the next section, this problem is closely related to the question of characterizing the polynomial solutions of the differential equation

$$
A(x) y^{\prime \prime}+2 B(x) y^{\prime}+C(x) y=0,
$$

where $A(x)=\left(x-a_{0}\right) \cdots\left(x-a_{p}\right), B(x)$ and $C(x)$ are polynomials of degree $p$ and $p-1$, and

$$
\frac{B(x)}{A(x)}=\sum_{j=0}^{p} \frac{r_{j}}{x-a_{j}} .
$$

This equation is called a Lamé equation in algebraic form. Heine proved that, given $A(x)$ and $B(x)$, there exist at most $(n+p-1) ! /(n !(p-1) !)$ polynomials $C(x)$, such that, for each of them, (1.1) has a polynomial solution $y(x)$ of degree $n$. Stieltjes proved that there are exactly $(n+p-1) ! /(n !(p-1) !)$ polynomials $C(x)$, such that, for each of them, there exists a polynomial solution $y(x)$ of degree $n$ with only real zeros of the above Lamé equation. Moreover, Stieltjes proved that each case corresponds to a distribution of the $n$ free charges in the $p$ intervals $\left(a_{j-1}, a_{j}\right), j=1, \ldots, p$. This result is known as the Heine-Stieltjes theorem [16, Theorem 6.8]. The polynomial $C(x)$ is called a Van Vleck polynomial and the corresponding polynomial solution $y(x)$ of (1.1) is called a Stieltjes polynomial. Van Vleck 18 was the first to prove that the zeros of $C(x)$ lie in $\left(a_{0}, a_{p}\right)$. Klein [7, Bôcher 2 and Pólya [10] proved that the zeros of Stieltjes polynomials lie in the convex hull of $a_{0}, \ldots, a_{p}$ if $a_{j}$ are complex provided the coefficients $r_{j}$ are positive. Marden [8] proved the same result under a weaker condition. He allowed the coefficients $r_{j}$ to be complex numbers with positive real parts. Alam [1] extended Marden's result. We refer to Chapter 2.9 of Marden's monograph [9] for more detailed information about Lamé's equation and to [19, 17] for the connection with electrostatics of zeros of orthogonal polynomials. In very recent papers Grünbaum 3] gave an electrostatic interpretation of the zeros of Koornwinder-Krall polynomials and Ismail [5] showed that the zeros of a general class of orthogonal polynomials are points of unique equilibrium in an electrostatic field and calculated the energy of the field. Grünbaum [4] established a result on electrostatics of zeros of the polynomials obtained from those of Jacobi by repeated applications of the Darboux transformation. Every application of the transformation introduces an additional parameter into the problem. It is interesting that the surface formed by the parameters has special points which are critical in the sense that the corresponding electrostatic problem becomes unstable and thus the system is not at equilibrium.

The electrostatic problem with both positive and negative charges has not been completely analyzed before; in particular the unicity of a Van Vleck polynomial 
(or the unicity of the equilibrium position) has not been obtained earlier. The first appearance of negative charges is in a recent paper by Grünbaum [3] who deals with a problem containing two negative charges between two positive charges, but he does not prove the unicity of the equilibrium. In this paper we prove the unicity of a Van Vleck polynomial $C(x)$ for certain situations involving both positive and negative coefficients $r_{j}$, which implies the existence and unicity of a Stieltjes polynomial $y(x)$ of (1.1). The zeros of $y(x)$ are located between two consecutive zeros $a_{j-1}$ and $a_{j}$ of $A(x)$ for which $r_{j-1}$ and $r_{j}$ are positive. As an application we prove that the zeros of Gegenbauer-Laurent polynomials are points of unique equilibrium in an electrostatic field generated by two positive and two negative charges.

\section{Electrostatics and Lamé's equation}

Consider an electrostatic field which obeys the logarithmic potential law and which is described as follows. Charges $r_{j}$ are distributed along long wires which are perpendicular to the real axes and intersect it at the points $a_{j}, j=0, \ldots, p$. The $n$ movable positive unit charges are also distributed along long wires which are perpendicular to the real axes and pass through the points $x_{1}, \ldots, x_{n}$. We require that the free charges move only between consecutive positive fixed charges in order to avoid coalescence. This means that the point $\left(x_{1}, \ldots, x_{n}\right)$ belongs to the simplex

$$
\Xi:=\bigcap_{\nu=1}^{s}\left\{a_{j_{\nu}}<x_{\mu_{\nu}}<\ldots x_{\mu_{\nu+1}-1}<a_{j_{\nu}+1}: r_{j_{\nu}}, r_{j_{\nu}+1}>0\right\}
$$

where $1=\mu_{1} \leq \mu_{2} \leq \cdots \mu_{s} \leq \mu_{s+1}-1=n$ and $s$ is the number of intervals $\left[a_{j_{\nu}}, a_{j_{\nu}+1}\right]$ for which there are positive charges $r_{j_{\nu}}$ and $r_{j_{\nu}+1}$ at the endpoints. The energy of the field is given by

$$
L\left(x_{1}, \ldots, x_{n}\right)=\sum_{j=0}^{p} r_{j} \sum_{k=1}^{n} \log \frac{1}{\left|a_{j}-x_{k}\right|}+\sum_{1 \leq i<k \leq n} \log \frac{1}{\left|x_{k}-x_{i}\right|} .
$$

It is clear that the energy is minimal if and only if the function

$$
T\left(x_{1}, \ldots, x_{n}\right):=\prod_{j=0}^{p} \prod_{k=1}^{n}\left|a_{j}-x_{k}\right|^{2 r_{j}} \prod_{1 \leq i<k \leq n}\left(x_{k}-x_{i}\right)^{2}
$$

is maximal. Observe that $T\left(x_{1}, \ldots, x_{n}\right)$ vanishes on the boundary of $\Xi$ and is strictly positive inside $\Xi$. Hence the maximum of $T$ is attained at a point $\left(x_{1}, \ldots, x_{n}\right)$ in $\Xi$ where $\partial T / \partial x_{k}=0$ for $k=1, \ldots, n$. On the other hand

$$
\begin{aligned}
T\left(x_{1}, \ldots, x_{n}\right) & =\tau\left(\bar{x} \backslash x_{k}\right) \prod_{j=0}^{p}\left|x_{k}-a_{j}\right|^{2 r_{j}} \prod_{i \neq k}\left(x_{k}-x_{i}\right)^{2} \\
& =\tau\left(\bar{x} \backslash x_{k}\right) \prod_{j=0}^{p}\left|x_{k}-a_{j}\right|^{2 r_{j}} \omega_{k}^{2}\left(x_{k}\right),
\end{aligned}
$$

where the function $\tau\left(\bar{x} \backslash x_{k}\right)$ does not depend on $x_{k}, \omega_{k}(x):=\omega(x) /\left(x-x_{k}\right)$ and $\omega(x):=\prod_{i=1}^{n}\left(x-x_{i}\right)$. 
Differentiating the latter identity and using the equalities $\omega^{(l+1)}\left(x_{k}\right)=$ $(l+1) \omega_{k}^{(l)}\left(x_{k}\right), l=0,1,2$, we obtain consecutively

$$
\begin{aligned}
\frac{\partial}{\partial x_{k}} T\left(x_{1}, \ldots, x_{n}\right)= & \tau\left(\bar{x} \backslash x_{k}\right) \frac{\partial}{\partial x_{k}}\left(\prod_{j=0}^{p}\left|x_{k}-a_{j}\right|^{2 r_{j}} \omega_{k}^{2}\left(x_{k}\right)\right) \\
= & \tau\left(\bar{x} \backslash x_{k}\right) \omega_{k}\left(x_{k}\right) \prod_{j=0}^{p}\left|x_{k}-a_{j}\right|^{2 r_{j}-1} \\
& \times 2 A\left(x_{k}\right)\left\{\omega_{k}^{\prime}\left(x_{k}\right)+\left(\sum_{j=0}^{p} r_{j} /\left(x_{k}-a_{j}\right)\right) \omega_{k}\left(x_{k}\right)\right\} \\
= & \tau\left(\bar{x} \backslash x_{k}\right) \omega_{k}\left(x_{k}\right) \prod_{j=0}^{p}\left(x_{k}-a_{j}\right)^{2 r_{j}-1} \\
& \times\left\{A\left(x_{k}\right) \omega^{\prime \prime}\left(x_{k}\right)+2 B\left(x_{k}\right) \omega^{\prime}\left(x_{k}\right)\right\} .
\end{aligned}
$$

The expression $A(x) \omega^{\prime \prime}(x)+2 B(x) \omega^{\prime}(x)$ is a polynomial of degree $n+p-1$ and at a maximum of $T$ this polynomial vanishes at the zeros of $\omega(x)$; hence

$$
A(x) \omega^{\prime \prime}(x)+2 B(x) \omega^{\prime}(x)+C(x) \omega(x)=0,
$$

for some polynomial $C(x)$ of degree $p-1$. Thus, if the energy of the electrostatic field under discussion has a unique point of minimum in $\Xi$, then there exists a unique pair $(C, y)$ of a Van Vleck and a Stieltjes polynomial for the Lamé equation (1.1). Conversely, if there exists a unique pair $(C, y)$ of a Van Vleck and a Stieltjes polynomial for (1.1), such that the zeros of the Stieltjes polynomial belong to $\Xi$, then the energy of the field has a unique minimum.

We will consider this electrostatic problem with both positive and negative charges. The following situations lead to a unique Van Vleck polynomial, and thus to a unique equilibrium.

Theorem 1. Let $A(x)=\left(x-a_{0}\right)\left(x-a_{1}\right)\left(x-a_{2}\right)\left(x-a_{3}\right), a_{0}<\cdots<a_{3}$, be a quartic and $B(x)$ be a cubic polynomial, for which the coefficients $r_{j-1}$ and $r_{j}$ in the partial fraction decomposition (1.2) are positive and the remaining two coefficients are negative. If

a) the sequence $r_{0}, r_{1}, r_{2}, r_{3}$ admits only one sign change, i.e., if $j=1$ or $j=3$, or

b) the sequence $r_{0}, r_{1}, r_{2}, r_{3}$ admits two sign changes, i.e., if $r_{0}<0, r_{1}>0, r_{2}>$ $0, r_{3}<0$, and $n>2+r_{0}+r_{1}+r_{2}+r_{3}$,

then there exists a unique pair $(C, y)$ with $C(x)$ a quadratic polynomial and $y(x)=$ $\left(x-x_{1}\right) \cdots\left(x-x_{n}\right)$ a solution of (1.1) for which $a_{j-1}<x_{1}<\cdots x_{n}<a_{j}$.

Proof. As it is seen from the discussion in Section 2, the existence of the Stieltjes polynomial with the desired properties is equivalent to the existence of a point of maximum $x^{*}=\left(x_{1}, \ldots, x_{n}\right)$ of $T\left(x_{1}, \ldots, x_{n}\right)$ in the simplex $\Xi:=\left\{a_{j-1}<x_{1}<\right.$ $\left.\ldots<x_{n}<a_{j}\right\}$. As was already mentioned, $T\left(x_{1}, \ldots, x_{n}\right)$ is strictly positive in $\Xi$ and vanishes on the boundary of $\Xi$ and thus such a point $x^{*}$ exists.

In order to establish the unicity, assume that there exists a polynomial $D(x)$ of degree $p-1, D(x) \not \equiv C(x)$, such that the differential equation

$$
A(x) z^{\prime \prime}+2 B(x) z^{\prime}+D(x) z=0
$$


also admits a solution $z(x)=\left(x-\xi_{1}\right) \cdots\left(x-\xi_{n}\right)$ with distinct zeros in $\left(a_{j-1}, a_{j}\right)$. Following Szegö [16, §6.83], we introduce the function $H(x)=\prod_{j=0}^{3}\left|x-a_{j}\right|^{2 r_{j}}$ and obtain, for $x \neq a_{\nu}, \nu=0, \ldots, 3$,

$$
\frac{d}{d x}\left\{H(x)\left(y^{\prime}(x) z(x)-y(x) z^{\prime}(x)\right)\right\}=\frac{D(x)-C(x)}{A(x)} y(x) z(x) H(x) .
$$

Set $x_{0}=\xi_{0}=a_{j-1}$ and $x_{n+1}=\xi_{n+1}=a_{j}$. A close inspection of Szegö's proof in the above mentioned $\S 6.83$ in [16] shows that:

1. If $(D(x)-C(x)) / A(x)$ is positive between two consecutive $x_{i}$ and $x_{i+1}$, with $0 \leq j \leq n$, then $z(x)$ must have a zero in $\left(x_{i}, x_{i+1}\right)$ and if $(D(x)-C(x)) / A(x)$ is negative between two consecutive $\xi_{i}$ and $\xi_{i+1}$, with $0 \leq i \leq n$, then $y(x)$ must have a zero in $\left(\xi_{i}, \xi_{i+1}\right)$.

2. $D(x)-C(x)$ must change sign at least once in $\left(a_{j-1}, a_{j}\right)$.

Since we may restrict ourselves to monic solutions of the Lamé equation, the leading coefficients of $D(x)$ and $C(x)$ are equal and then $D(x)-C(x)$ is a linear function. In order to prove the unicity of the Van Vleck polynomial, we shall show that $D(x)-C(x)$ has at least one more zero, which is only possible if $C \equiv D$. Assume the contrary; then by 2) $D(x)-C(x)$ has a unique zero in $\left(a_{j-1}, a_{j}\right)$ and by 1$)$ we can conclude that the zeros of $y(x)$ and $z(x)$ interlace. Therefore the coefficients $\delta_{k}$ in the decomposition

$$
\frac{y(x)}{z(x)}=1+\sum_{k=1}^{n} \frac{\delta_{k}}{x-\xi_{k}}
$$

have all the same sign. On the other hand,

$$
H(x)\left(y^{\prime}(x) z(x)-y(x) z^{\prime}(x)\right)=H(x) z^{2}(x)\left(\frac{y(x)}{z(x)}\right)^{\prime} .
$$

Since obviously

$$
\left(\frac{y(x)}{z(x)}\right)^{\prime}=-\sum_{k=1}^{n} \frac{\delta_{k}}{\left(x-\xi_{k}\right)^{2}},
$$

then

$$
H(x)\left(y^{\prime}(x) z(x)-y(x) z^{\prime}(x)\right)=-H(x) \sum_{k=1}^{n} \delta_{k} z_{k}^{2}(x),
$$

where $z_{k}(x)=z(x) /\left(x-\xi_{k}\right)$.

Suppose that all $\delta_{k}$ are positive (the case where all $\delta_{k}$ are negative can be handled in a similar way). Then the function $G(x)=-H(x) \sum_{k=1}^{n} \delta_{k} z_{k}^{2}(x)$ is non-positive, vanishes only at the zeros of $H(x)$, and converges to $-\infty$ at the poles of $H(x)$.

Consider first the case a). Except for the two consecutive zeros $a_{j-1}$ and $a_{j}$ of $H(x)$, we have also two consecutive poles. Then the derivative of $G(x)$ must change its sign in the interval between the poles of $H(x)$ since $G(x)$ will have a maximum between these two poles. In view of (2.3), this gives the desired additional zero of $D(x)-C(x)$ and proves the unicity in this case.

The requirement in b) is equivalent to the fact that $G(x)$ converges to $-\infty$ as $x$ approaches $-\infty$ or $+\infty$. Since $a_{0}$ and $a_{3}$ are poles of $H(x)$, this means that $G(x)$ will have a maximum between $-\infty$ and $a_{0}$ and also between $a_{3}$ and $+\infty$. Thus we even have two additional zeros of the derivative of $G(x)$, which, in view of (2.3), provide two more zeros of $D(x)-C(x)$. 


\section{Gegenbauer-Laurent polynomials AND ELECTROSTATIC INTERPRETATION OF THEIR ZEROS}

The distribution $d \psi(x)$ is said to be a strong distribution in $(a, b) \subset(0, \infty)$ if $\psi(x)$ is a real bounded nondecreasing function on $(a, b)$ with infinitely many points of increase there, and furthermore, all the moments

$$
\mu_{k}=\int_{a}^{b} x^{k} d \psi(x), \quad k=0, \pm 1, \pm 2, \ldots
$$

exist.

For any given strong distribution in $(a, b)$ there exists a unique, up to a nonzero constant normalizing factor, sequence of polynomials $\left\{B_{n}\right\}_{0}^{\infty}$ such that $B_{n}$ is a polynomial of precise degree $n$ and $B_{n}$ satisfies the relations

$$
\int_{a}^{b} x^{-n+k} B_{n}(x) d \psi(x)=0, \quad k=0, \ldots, n-1 .
$$

The first systematic study of these polynomials, which may be called orthogonal Laurent polynomials or simply orthogonal L-polynomials, was done by Jones, Thron and Waadeland [6] in connection with the so-called strong Stieltjes moment problem. Many interesting properties of orthogonal Laurent polynomials are proven in 6. We are particularly interested in the behavior of the zeros of $B_{n}$. Their location is similar as in the case of orthogonal polynomials. All the zeros of $B_{n}$ are real, distinct and lie in $(a, b)$.

Recently Sri Ranga [12] provided a ingenious way of obtaining sequences of orthogonal Laurent polynomials by a change of variables in a sequence $\left\{p_{n}(t)\right\}$ of orthogonal polynomials with respect to an even weight function on a symmetric interval $(-d, d)$. Consider the classical Gegenbauer (ultraspherical) polynomials $C_{n}^{(\lambda)}(t)$, orthogonal on $[-1,1]$ with respect to the weight function $\left(1-t^{2}\right)^{\lambda-1 / 2}$. Sri Ranga proved that the polynomials

$$
B_{n}^{\lambda}(x)=C_{n}^{\lambda}(t)
$$

where

$$
t=\frac{1}{\sqrt{b}-\sqrt{a}} \frac{x-\sqrt{a b}}{\sqrt{x}}
$$

are orthogonal Laurent polynomials on $(a, b), 0<a<b$, with respect to the strong distribution $d \psi(x)=x^{-\lambda}(b-x)^{\lambda-1 / 2}(x-a)^{\lambda-1 / 2} d x$. Performing the above change of variables in the well-known second order differential equation (cf. (4.7.5) on page 80 in [16]) satisfied by the Gegenbauer polynomials, we conclude that $y(x)=B_{n}^{\lambda}(x)$ is a solution of the differential equation of the form (1.1) with

$$
\begin{aligned}
A(x)= & 2 x(b-x)(x-a)(x+\sqrt{a b}), \\
B(x)= & (n-\lambda-1) x^{3}-((n-1 / 2)(a+b)-(n-\lambda-2) \sqrt{a b}) x^{2} \\
& -((n-3 / 2)(a+b) \sqrt{a b}-(n+\lambda) a b) x+(n+\lambda-1)(a b)^{3 / 2}, \\
C(x)= & 2 \lambda n x^{2}+n((n+4 \lambda+1) \sqrt{a b}+(n+1)(a+b) / 2) x \\
& +n(n+2 \lambda-1) a b+n(n-1)(a+b) \sqrt{a b} / 2 .
\end{aligned}
$$


Then the partial fraction decomposition of $B(x) / A(x)$ is

$$
\frac{B(x)}{A(x)}=\frac{2 \lambda+1}{4} \frac{1}{x-a}+\frac{2 \lambda+1}{4} \frac{1}{x-b}-\frac{n+\lambda-1}{2} \frac{1}{x}-\frac{1}{2} \frac{1}{x+\sqrt{a b}} .
$$

Now Theorem 1 a) immediately implies:

Theorem 2. Consider the electrostatic field, which obeys the logarithmic potential law and is generated by two positive charges of common value $(2 \lambda+1) / 4$ at the points $a$ and $b, 0<a<b$, and negative charges $-1 / 2$ at the point $-\sqrt{a b}$ and $-(n+\lambda-1) / 2$ at the origin. Then the zeros of the Gegenbauer-Laurent polynomial $B_{n}^{\lambda}(x)$ are the points of unique equilibrium of $n$ movable charges in $(a, b)$.

The two negative charges at $-\sqrt{a b}$ and at the origin are both to the left of the interval $[a, b]$ and attract the zeros of $B_{n}^{\lambda}(x)$. This means that the zeros are more concentrated near $a$ than near $b$ and their asymptotic distribution is not the arcsin distribution on $[a, b]$. In fact, Sri Ranga [12] proved that the zeros of $B_{n}^{\lambda}(x)$ are 'symmetric' with respect to $\sqrt{a b}$ in the following sense: if $x_{k}$ is a zero, then $a b / x_{k}$ is also a zero.

\section{REFERENCES}

[1] M. Alam, Zeros of Stieltjes and Van Vleck polynomials, Trans. Amer. Math. Soc. 252 (1979), 197-204. MR 81g:30011

[2] M. Bôcher, The roots of polynomials that satisfy certain differential equations of the second order, Bull. Amer. Math. Soc. 4 (1897), 256-258.

[3] F. A. Grünbaum, Variations on a theme of Heine and Stieltjes: an electrostatic interpretation of the zeros of certain polynomials, J. Comput. Appl. Math. 99 (1998), 189-194. MR 99j:33012

[4] F. A. Grünbaum, Electrostatics and the Darboux process, in preparation.

[5] M. E. H. Ismail, An electrostatics model for zeros of general orthogonal polynomials, Pacific J. Math., to appear in 1999.

[6] W. B. Jones, W. J. Thron and H. Waadeland, A strong Stieltjes moment problem, Trans. Amer. Math. Soc. 261 (1980), 503-528. MR 81j:30055

[7] F. Klein, Über die Nullstellen von den Polynomen und den Potenzreihen, Göttingen, 1894, pp. 211-218.

[8] M. Marden, On Stieltjes polynomials, Trans. Amer. Math. Soc. 33 (1931), 934-944.

[9] M. Marden, Geometry of Polynomials, Amer. Math. Soc. Surveys, no. 3, Providence, R.I., 1966. MR 37:1562

[10] G. Pólya, Sur un théorème de Stieltjes, C. R. Acad. Sci. Paris 155 (1912), 767-769.

[11] E. M. Purcell, Electricity and Magnetism, Berkeley Physics Course - Volume 2, McGrawHill, New York, 1963.

[12] A. Sri Ranga, Symmetric orthogonal polynomials and the associated orthogonal L-polynomials, Proc. Amer. Math. Soc. 123 (1995), 3135-3141. MR 95m:42035

[13] T. J. Stieltjes, Sur les quelques théorémes d'algébre, C.R. Acad. Sci. Paris 100 (1885), 439440.

[14] T. J. Stieltjes, Sur les polynômes de Jacobi, C.R. Acad. Sci. Paris 100 (1885), 620-622.

[15] T. J. Stieltjes, Sur les racines de l'équation $X_{n}=0$, Acta Math. 9 (1886), 385-400.

[16] G. Szegö, Orthogonal polynomials, 4th ed., Amer. Math. Soc. Coll. Publ., Vol. 23, Providence, RI, 1975. MR 51:8724

[17] G. Valent and W. Van Assche, The impact of Stieltjes' work on continued fraction and orthogonal polynomials: additional material, J. Comput. Appl. Math. 65 (1995), 419-447. MR 97c:33001 
[18] E. B. Van Vleck, On the polynomials of Stieltjes, Bull. Amer. Math. Soc. 4 (1898), 426-438.

[19] W. Van Assche, The impact of Stieltjes' work on continued fraction and orthogonal polynomials, in "T. J. Stieltjes: Collected papers, Vol. I" (G. van Dijk, ed.), pp. 5-37, Springer-Verlag, Berlin, 1993.

Departamento de Ciências de Computação e Estatística, ibilce, Universidade Estadual Paulista, 15054-000 São José do Rio Preto, SP, Brazil

E-mail address: dimitrov@nimitz.dcce.ibilce.unesp.br

Department of Mathematics, Katholieke Universiteit Leuven, Celestijnenlaan 200

B, B-3001 Heverlee (Leuven), Belgium

E-mail address: Walter.VanAssche@wis.kuleuven.ac.be 